\title{
An evaluation of obstetric ultrasound education program in Nepal using the RE- AIM framework
}

Jieun Kim ${ }^{1 \dagger}$, Prabin Raj Shakya ${ }^{1,2 \dagger}$, Sugy Choi ${ }^{1,3}$, Joong Shin Park ${ }^{1,4}$, Suman Raj Tamrakar ${ }^{5}$, Jongho Heo ${ }^{1,6^{*}}$ and Woong-Han Kim ${ }^{1,7^{*}}$

\begin{abstract}
Background: Nepal has a high prevalence of congenital anomaly contributing to high infant mortality. Ultrasound, an important tool to detect congenital anomalies and manage maternity-related risk factors, is not properly used in Nepal because Nepali doctors have limited opportunities for learning ultrasound techniques. Hence, we developed and implemented an ultrasound education program from 2016 to 2018. The objective of this study is to evaluate the education program using the Reach, Effectiveness, Adoption, Implementation, and Maintenance (RE-AIM) framework.

Methods: We conducted a mixed-method study to evaluate each component of RE-AIM. The team collected quantitative data from administrative records, tests, surveys, and an online follow-up survey. Qualitative data were collected from individual in-depth interviews at least a year after the program. The proportions, means, and t-tests were used for quantitative data, and thematic coding for qualitative data.

Results: A total of 228 healthcare workers representing 27.3\% of the districts of Nepal were reached from 2016 to 2018. The program improved participants' knowledge (29.3, 8.7, and 23.8 increases out of 100, each year, $p<0.001$, $n=85)$ and self-confidence $(0.6,0.3,1.3$ increases out of $4.0, p<0.01, n=111)$. The participants were highly satisfied with the program $(4.2,4.1$, and 4.0 out of 5.0, $n=162)$. Among the respondents of the online follow-up survey $(n=$ 28), $60.7 \%$ had used ultrasound in their daily practice after the education program, and a medical institution established an ultrasound training center. The absence of clear accreditation and practical guidelines in ultrasound use were presented as barriers for adoption and maintenance.

Conclusion: The program was successful in improving participant's knowledge and self-confidence in ultrasound techniques and showed great potential for the adoption and maintenance of the techniques in their practice. Continuous implementation of the program and institutional policy changes to facilitate ultrasound use may increase the ultrasound use and improve ultrasound service quality in Nepal.
\end{abstract}

Keywords: Obstetric ultrasound, Education, Program evaluation, RE-AIM, LMIC, Global education

\footnotetext{
*Correspondence: joheo@nafi.re.kr; woonghan@snu.ac.kr

†Jieun Kim and Prabin Raj Shakya are co-first authors and contributed equally to this work.

'JW LEE Center for Global Medicine, Seoul National University College of Medicine, Seoul, Republic of Korea

Full list of author information is available at the end of the article
}

(C) The Author(s). 2021 Open Access This article is licensed under a Creative Commons Attribution 4.0 International License, which permits use, sharing, adaptation, distribution and reproduction in any medium or format, as long as you give appropriate credit to the original author(s) and the source, provide a link to the Creative Commons licence, and indicate if changes were made. The images or other third party material in this article are included in the article's Creative Commons. licence, unless indicated otherwise in a credit line to the material. If material is not included in the article's Creative Commons licence and your intended use is not permitted by statutory regulation or exceeds the permitted use, you will need to obtain permission directly from the copyright holder. To view a copy of this licence, visit http://creativecommons.org/licenses/by/4.0/ The Creative Commons Public Domain Dedication waiver (http://creativecommons.org/publicdomain/zero/1.0/) applies to the data made available in this article, unless otherwise stated in a credit line to the data. 


\section{Background}

Congenital anomaly contributes to $5-7 \%$ of perinatal and neonatal mortality in low- and middle-income countries (LMICs) [1]. The prevalence of congenital anomaly in Nepal is also high (52 per 10,000 children), and 11.1\% of neonates die because of congenital anomalies [1-3]. Yet, literature on the care and management of defects in Nepal is limited. Birth defects is an emerging public health problem due to lack of genetic diagnostic methods and inadequacy of other identification methods [4]. Ultrasound is an important noninvasive screening tool that detects congenital anomalies and manages maternity-related risk factors for both morbidity and mortality $[5,6]$. The World Health Organization (WHO) recommends pregnant women undergo at least one ultrasound scan before 24 weeks of gestation [7].

Ultrasound has become more widely available for medical professionals in LMICs [8] with decreased equipment costs. However, challenges in improving ultrasound service quantity and quality remain. Workforce shortage is considered a major challenge and a few opportunities for ultrasound training and continuing education exacerbate this challenge. Moreover, several ultrasound training in LMICs does not meet the WHO standards [9].

These challenges are also evident in Nepal. In Nepal, radiologists generally perform ultrasound scans, but Nepal has only 150 radiologists (1 per 185,000 populations), who are largely concentrated around the capital city [10]. This uneven distribution overwhelms ultrasound services in urban areas and thus, service quality control in antenatal care is difficult to achieve [10-12]. Moreover, non-radiologists have limited ultrasound training during their medical school, engaging only a short 15-day rotation in the radiology department. Hence, their ultrasound skill is either basic (limited to acquiring an idea on how machines operate and performing a few procedures with low-quality images) or poor $[11,13]$.

Previous studies show that intensive ultrasound training courses in LMICs significantly increase knowledge and self-confidence among the radiologists [13, 14]. Hence, the JW LEE Center for Global Medicine at Seoul National University College of Medicine (JW LEE CGM) of the Republic of Korea initiated the intensive ultrasound education program in Nepal since 2016 to promote ultrasound utilization and improve the quality of the practice among both radiologists and nonradiologists through continuous education and experience sharing. In this study, we evaluated the impacts of this education program from 2016 to 2018 using the Reach, Effectiveness, Adoption, Implementation, and Maintenance (RE-AIM) framework [15]. The framework includes an explicit focus on issues, dimensions, and steps in the program design, dissemination, and implementation process, which had been translated and used in different contexts, populations, settings, and cultures [15-18]. We applied the framework to evaluate the effectiveness of knowledge translation, assess broader impacts, and comprehensively understand the limitations, using quantitative and qualitative methods.

\section{Methods \\ Education program development}

JW LEE CGM held multiple rounds of needs assessments with key experts from Nepal. Respondents expressed multiple topics of interest such as cutting edge technology in medicine, such as Computed Tomography (CT) and Magnetic Resonance Imaging (MRI); fetal health screening for different systems using ultrasound, CT, and MRI; CT and MRI image and report interpretation; recent developments and issues in ultrasound techniques; detailed demonstration on biometry and other important indicator calculations using different imaging techniques. After exploring the perceived needs, JW LEE CGM invited lecturers/experts, Dhulikhel Hospital-Kathmandu University Hospital (DHKUH), and Nepali partners for educational purposes and created the curriculum based on the needs and availability of lecturers. The course was for two days and titled "The Ultrasound, CT and MRI Education Program for Obstetric, Fetal, Neonatal, and Congenital Anomaly" The course was conducted in collaboration with the Nepali Professional Societies. Table 1 shows the program agenda in 2016, 2017, and 2018. The contents were arranged based on feedback from the previous year's participants and experts involved (obstetrician-gynecologists [OB-GYNs], pediatricians, radiologists, and hospital leaders). The core component of the program included lectures on obstetric image technology and techniques, case discussions, and experience sharing from both the Korean and Nepali experts. The components of CT and MRI in the program were considered as supplementary sessions because most of the hospitals in Nepal do not perform CT and MRI [19].

\section{Participant recruitment for the education program}

A national open call for recruitment in the education program was made through various media and professional societies. The main target group of the program was OB-GYNs, pediatricians, and radiologists, but medical doctors from different specialties and non-physician healthcare providers (e.g., nurses, midwives, and paramedical staff) were also recruited. A total of 228 healthcare workers participated in the education program from 2016 to 2018. 
Table 1 Program agenda of ultrasound, CT, and MRI educational program for obstetric, fetal, neonatal and congenital anomaly in Nepal, 2016, 2017, and 2018

\begin{tabular}{|c|c|c|}
\hline 2016 & 2017 & 2018 \\
\hline \multicolumn{3}{|l|}{ Day 1 Sessions } \\
\hline Basic fetal echocardiography & Fetal CNS & Obstetric USG \\
\hline $\begin{array}{l}\text { Fetal cardiac physiology and transitional } \\
\text { circulation }\end{array}$ & Fetal thorax & Biometry and amniotic fluid volume \\
\hline $\begin{array}{l}\text { Cardiac CT before congenital heart disease } \\
\text { surgery }\end{array}$ & Fetal abdomen & Fetal abdomen \\
\hline $\begin{array}{l}\text { Cardiac MRI for congenital heart disease: } \\
\text { when? }\end{array}$ & Genetic sonography & Fetal genitourinary tract \\
\hline Fetal CNS & $\begin{array}{l}\text { Cervical length and prevention of preterm } \\
\text { birth }\end{array}$ & Fetal echocardiography for congenital heart disease \\
\hline Doppler USG in obstetrics & Dhulikhel Hospital USG experience & Pediatric echocardiography for congenital heart disease \\
\hline $\begin{array}{l}\text { Experience of anomaly scan in Dhulikhel } \\
\text { Hospital }\end{array}$ & $\begin{array}{l}\text { Live-demonstration: fetal screening } \\
\text { ultrasound }\end{array}$ & Dhulikhel Hospital experience in nuchal translucency \\
\hline $\begin{array}{l}\text { Live-demonstration: fetal and neonatal cardiac } \\
\text { ultrasound }\end{array}$ & $\begin{array}{l}\text { Live-demonstration: fetal Doppler } \\
\text { ultrasound }\end{array}$ & $\mathrm{CT}$ Interpretation of complex congenital heart disease \\
\hline Live-demonstration: fetal Doppler ultrasound & & $\begin{array}{l}\text { CT interpretation: extracardiac evaluation in congenital } \\
\text { heart disease }\end{array}$ \\
\hline \multicolumn{3}{|l|}{ Live-demonstration: Fetal screening ultrasound } \\
\hline \multicolumn{3}{|l|}{ Day 2 Sessions } \\
\hline Obstetric USG & Fetal VSD, AVSD & Fetal CNS \\
\hline Ultrasound of multiple gestation & TOF, pulmonary atresia, TGA & Fetal thorax \\
\hline Biometry and amniotic fluid volume & $\begin{array}{l}\mathrm{CT} \text { interpretation of complex congenital } \\
\text { heart disease }\end{array}$ & Fetal Doppler ultrasound \\
\hline $\begin{array}{l}\text { Experience nuchal translucency in Dhulikhel } \\
\text { Hospital }\end{array}$ & $\begin{array}{l}\text { Radiologic evaluation: adult congenital } \\
\text { heart disease }\end{array}$ & Genetic sonography \\
\hline
\end{tabular}

\section{Program evaluation framework}

This study utilized the RE-AIM framework, which is a frequently used tool that evaluates multiorganizational and country projects [16]. The RE-AIM framework was applied as a conceptual guide for the study to describe the evaluation process systematically and comprehensively. This framework was selected as it is the most frequently used, and it has been tested with a wide range of interventions. The framework puts special attention on the implementation context [16]. The "Reach" dimension defines the beneficiary population and presents it in numbers covered with demographic and professional characteristics. "Effectiveness" as an impact measurement of an intervention on outcomes, "Adoption" reports settings of intervention agents can be qualitative or quantitative, and "Implementation" describes implementation context and factors influencing implementation. "Maintenance" reports the sustainability of the intervention and factors leading to sustainment. The detailed definitions, indicators, and data sources are presented in Table 2.

\section{Data collection and outcomes}

This study used both quantitative and qualitative methods for evaluation. Administrative data, test data and survey data among participants were collected during the education program (2016-2018), and an online follow-up survey was conducted in 2019. Two members of the research team conducted face-to-face, in-depth interviews in English with the participants and leaders of participating institutions $(n=11)$. The interviewees were recruited using convenience sampling.

Outcomes of the study were used in accordance with the components of the RE-AIM framework-Reach, Effectiveness, Adoption, Implementation, and Maintenance. Table 2 shows the details on the process and outcome indicators along with the utilized data sources.

\section{Analysis}

This study used proportions and means for the descriptive analysis and used the mean comparison t-test for difference in pre- and post-test scores for knowledge and pre- and post-survey scores for self-confidence. All quantitative data were analyzed using Stata version 14. 
Table 2 Indicators and data sources for evaluating process and outcomes according to the reach, effectiveness, adoption, implementation, and maintenance framework

\begin{tabular}{|c|c|c|c|}
\hline Dimensions & Definitions & Indicators & $\begin{array}{l}\text { Data Sources } \\
\text { (Tools) }\end{array}$ \\
\hline Reach & $\begin{array}{l}\text { An individual-level measure that describes the population the } \\
\text { intervention intended to benefit and those that participated or } \\
\text { were exposed to the intervention. It is reported as the absolute } \\
\text { number, proportion, and representativeness of individuals. } \\
(\text { Glasgow }(1999,2018))\end{array}$ & $\begin{array}{l}\text { Yearly description of participants by } \\
\text { demographic characteristics, professional } \\
\text { characteristics and geographic coverage. }\end{array}$ & $\begin{array}{l}\text { Enrollment } \\
\text { records, initial } \\
\text { paper survey }\end{array}$ \\
\hline \multirow[t]{3}{*}{ Effectiveness } & \multirow{3}{*}{$\begin{array}{l}\text { The impact measurement of an intervention on outcomes, } \\
\text { including both positive and negative effects. It is presented as } \\
\text { an average of the overall effect of outcomes. }\end{array}$} & $\begin{array}{l}\text { Mean difference scores in pre- and post-levels } \\
\text { of knowledge }\end{array}$ & $\begin{array}{l}\text { Pre- and post- } \\
\text { test }\end{array}$ \\
\hline & & $\begin{array}{l}\text { Mean difference scores in pre- and post-levels } \\
\text { of self-confidence }\end{array}$ & $\begin{array}{l}\text { Pre- and post- } \\
\text { survey }\end{array}$ \\
\hline & & $\begin{array}{l}\text { Satisfaction scores of healthcare providers } \\
\text { who participated the education program }\end{array}$ & Paper survey \\
\hline \multirow{3}{*}{$\begin{array}{l}\text { Adoption } \\
\text { (Individual } \\
\text { Level) }\end{array}$} & \multirow{3}{*}{$\begin{array}{l}\text { Reports settings of a program or policy and intervention } \\
\text { agents. This dimension can be reported as a rate or proportion } \\
\text { but not limited to those, and qualitative measures can be used. }\end{array}$} & $\begin{array}{l}\text { Percentage of participants reporting the use } \\
\text { of the techniques learned }\end{array}$ & $\begin{array}{l}\text { Online survey } \\
\text { via email }\end{array}$ \\
\hline & & Techniques used in current practice & \multirow{2}{*}{$\begin{array}{l}\text { In-depth } \\
\text { interview }\end{array}$} \\
\hline & & $\begin{array}{l}\text { What were the participants' attitudes and } \\
\text { beliefs toward the intervention? }\end{array}$ & \\
\hline \multirow[t]{3}{*}{ Implementation } & \multirow{3}{*}{$\begin{array}{l}\text { Describes how the program or policy is delivered, the } \\
\text { economics behind a program or policy, and the } \\
\text { implementation factors associated with results. The studies' } \\
\text { context implementation dimension focuses on strategies and } \\
\text { qualitative approaches used to understand the reasons behind } \\
\text { the outcomes. }\end{array}$} & $\begin{array}{l}\text { Description of implementation strategy and } \\
\text { processes }\end{array}$ & Protocol \\
\hline & & Implementing partners & Administrative \\
\hline & & $\begin{array}{l}\text { Inclusion of all components of education } \\
\text { program. (Fig. 1) }\end{array}$ & records \\
\hline \multirow{2}{*}{$\begin{array}{l}\text { Maintenance } \\
\text { (Organizational } \\
\text { Level) }\end{array}$} & \multirow{2}{*}{$\begin{array}{l}\text { Addresses operational times, the sustainability of the } \\
\text { intervention, and factors leading to sustainment. It is reported } \\
\text { as a rate or proportion of the recipients that sustained the } \\
\text { intervention and institutionalization of practice or policy. } \\
\text { Qualitative approaches are also adopted to understand multi- } \\
\text { level factors related to sustainment. }\end{array}$} & $\begin{array}{l}\text { Policies and Infrastructure to ensure long-term } \\
\text { use of ultrasound }\end{array}$ & \multirow[t]{2}{*}{$\begin{array}{l}\text { In-depth } \\
\text { interview }\end{array}$} \\
\hline & & $\begin{array}{l}\text { Initiatives from partners in Nepal which } \\
\text { sustains education program }\end{array}$ & \\
\hline
\end{tabular}

For the qualitative data, voice recordings were transcribed and thematically coded using a software R Qualitative Data Analysis (RQDA) package for analysis [20]. PRS coded and formulated the themes based on the inductive method [21], and JK reviewed the codes. Both PRS and JK iteratively refined the subthemes (conceptual labels) and themes (code categories) and linked these with the RE-AIM framework dimensions.

\section{Ethics}

Regarding the tests and surveys, participants verbally consented and voluntarily participated. Written consent was obtained for the online survey and in-depth interviews. Ethical approval was obtained from Institutional Review Boards at the Seoul National University Hospital in the Republic of Korea (SNUH IRB No. 1906-036-1038).

\section{Results}

Reach

Table 3 summarizes the demographic characteristics of all participants in the ultrasound education program from 2016 to 2018. Across all years, a total of 228 healthcare workers attended the program, and most of them were aged less than 30 years (46.5, 41.3, and $75.8 \%$ in 2016, 2017, and 2018, respectively) and were affiliated with medical colleges $(70.7,54.0$, and $81.8 \%$ in 2016, 2017, and 2018, respectively), but participants representing other types of institutions such as private hospital, government hospital, healthcare center, and NonGovernmental Organization (NGO) hospital were also present. In case of specialty, all the prioritized groups (OB-GYNs, pediatricians, and radiologists) had similar representation across all 3 years. In terms of geographic coverage calculated by the proportion of districts where the participants' institution was located (Fig. 2) over the whole districts, one-third of the districts of Nepal $(27.3 \%$ in 3 years, $16.9 \%$ in $2016,12.9 \%$ in 2017 , and $10.4 \%$ in 2018) were reached. Most of the participating institutes were from capital and its vicinity.

\section{Effectiveness}

Table 4 indicates the changes in knowledge and selfconfidence before and after the education and overall satisfaction of the program. The mean post-test scores were 78.2, 50.1 and 59.3 out of 100 from 2016 to 2018 . The test scores were significantly increased by $29.3,8.7$, and $23.8(p<0.001)$ compared to pre-test scores. 
Table 3 Characteristics of the participants (2016 to 2018) and online follow-up survey respondents (2019)

\begin{tabular}{|c|c|c|c|c|c|c|c|}
\hline Characteristics & & 2016 & 2017 & 2018 & $\begin{array}{l}\text { Total Participants (2016 to } \\
\text { 2018) }\end{array}$ & $\begin{array}{l}\text { Online Follow-Up Survey Respondents } \\
\text { (2019) }\end{array}$ & $\begin{array}{l}P \text { - } \\
\text { Value }\end{array}$ \\
\hline Total N (\%) & & $\begin{array}{l}99 \\
(100)\end{array}$ & $\begin{array}{l}63 \\
(100)\end{array}$ & $\begin{array}{l}66 \\
(100)\end{array}$ & $228(100)$ & $28(100)$ & \\
\hline \multirow[t]{2}{*}{ Sex } & Male & $\begin{array}{l}65 \\
(65.7)\end{array}$ & $\begin{array}{l}43 \\
(68.3)\end{array}$ & $\begin{array}{l}26 \\
(39.4)\end{array}$ & $134(58.8)$ & $22(78.6)$ & 0.068 \\
\hline & Female & $\begin{array}{l}34 \\
(34.3)\end{array}$ & $\begin{array}{l}20 \\
(31.8)\end{array}$ & $\begin{array}{l}40 \\
(60.6)\end{array}$ & $94(41.2)$ & $6(21.4)$ & \\
\hline \multirow[t]{4}{*}{ Age } & $18-29$ & $\begin{array}{l}46 \\
(46.5)\end{array}$ & $\begin{array}{l}26 \\
(41.3)\end{array}$ & $\begin{array}{l}50 \\
(75.8)\end{array}$ & $122(53.5)$ & $10(35.7)$ & $<0.001$ \\
\hline & $30-39$ & $\begin{array}{l}37 \\
(37.4)\end{array}$ & $\begin{array}{l}25 \\
(39.7)\end{array}$ & $\begin{array}{l}10 \\
(15.2)\end{array}$ & $72(31.6)$ & $3(10.7)$ & \\
\hline & $40+$ & $\begin{array}{l}14 \\
(14.1)\end{array}$ & $\begin{array}{l}10 \\
(15.9)\end{array}$ & $5(7.6)$ & $29(12.7)$ & $14(50)$ & \\
\hline & Missing & $2(2.0)$ & $2(3.2)$ & $1(1.5)$ & $5(2.2)$ & $1(3.6)$ & \\
\hline \multirow[t]{3}{*}{$\begin{array}{l}\text { Type of } \\
\text { Institution }\end{array}$} & $\begin{array}{l}\text { Medical } \\
\text { college }\end{array}$ & $\begin{array}{l}70 \\
(70.7)\end{array}$ & $\begin{array}{l}34 \\
(54.0)\end{array}$ & $\begin{array}{l}54 \\
(81.8)\end{array}$ & $158(69.3)$ & $14(50)$ & 0.039 \\
\hline & Others $^{*}$ & $\begin{array}{l}26 \\
(26.3)\end{array}$ & $\begin{array}{l}29 \\
(46.0)\end{array}$ & $9(13.6)$ & $64(28.1)$ & $14(50)$ & \\
\hline & Missing & $3(3.0)$ & $0(0.0)$ & $3(4.6)$ & $6(2.6)$ & 0 & \\
\hline \multirow[t]{5}{*}{ Specialty } & OB-GYN***** & $\begin{array}{l}26 \\
(26.3)\end{array}$ & $\begin{array}{l}12 \\
(19.1)\end{array}$ & $\begin{array}{l}12 \\
(18.2)\end{array}$ & $50(21.9)$ & $8(28.6)$ & \\
\hline & Pediatrician & $\begin{array}{l}16 \\
(16.2)\end{array}$ & $7(11.1)$ & $6(9.1)$ & $29(12.7)$ & $2(7.1)$ & 0.762 \\
\hline & Radiologist & $\begin{array}{l}19 \\
(19.2)\end{array}$ & $\begin{array}{l}13 \\
(20.6)\end{array}$ & $4(6.1)$ & $36(15.8)$ & $4(14.3)$ & \\
\hline & Others $^{* *}$ & $\begin{array}{l}38 \\
(38.4)\end{array}$ & $\begin{array}{l}31 \\
(49.2)\end{array}$ & $\begin{array}{l}41 \\
(62.1)\end{array}$ & $110(48.2)$ & $14(50)$ & \\
\hline & Missing & $0(0.0)$ & $0(0.0)$ & $3(4.6)$ & $3(1.3)$ & 0 & \\
\hline \multirow[t]{3}{*}{ Experience } & $<3$ years & $\begin{array}{l}29 \\
(29.3)\end{array}$ & $\begin{array}{l}44 \\
(69.8)\end{array}$ & $\begin{array}{l}38 \\
(57.6)\end{array}$ & $111(48.7)$ & $14(50.0)$ & 0.179 \\
\hline & $\geq 3$ years & $\begin{array}{l}24 \\
(24.2)\end{array}$ & $\begin{array}{l}18 \\
(28.6)\end{array}$ & $\begin{array}{l}17 \\
(25.8)\end{array}$ & 59 (25.9) & $14(50.0)$ & \\
\hline & Missing & $\begin{array}{l}46 \\
(46.5)\end{array}$ & $1(1.6)$ & $\begin{array}{l}11 \\
(16.7)\end{array}$ & $58(25.4)$ & 0 & \\
\hline \multirow[t]{4}{*}{ Position } & Junior & $\begin{array}{l}59 \\
(59.6)\end{array}$ & $\begin{array}{l}31 \\
(49.2)\end{array}$ & $\begin{array}{l}45 \\
(68.2)\end{array}$ & $135(59.2)$ & $50(50.0)$ & $<0.001$ \\
\hline & Senior & $\begin{array}{l}29 \\
(29.3)\end{array}$ & $\begin{array}{l}17 \\
(27.0)\end{array}$ & $7(10.6)$ & $53(23.2)$ & $50(50.0)$ & \\
\hline & Others $^{* * *}$ & $9(9.1)$ & $\begin{array}{l}14 \\
(22.2)\end{array}$ & $\begin{array}{l}13 \\
(19.7)\end{array}$ & $36(15.8)$ & 0 & \\
\hline & Missing & $2(2.0)$ & $1(1.6)$ & $1(1.5)$ & $4(1.8)$ & 0 & \\
\hline
\end{tabular}

Note: "Private hospital and clinics, non-governmental organization hospital, government health institutions ${ }^{* *}$ Emergency, nursing, surgery

${ }^{* * *}$ Director

${ }^{* * * *} P$-value for chi-squared test of independence between the total participants and online survey population

${ }^{* * * * *} \mathrm{OB}-\mathrm{GYN}$ : obstetrician-gynecologist

Similarly, the mean post-self-confidence scores were 3.0 in all three years which were increased significantly by $0.6,0.3$, and 1.3 out of 4 points $(p<0.01)$ compared to pre-self-confidence scores. The participant's overall satisfaction score of the education program was high (4.2, 4.1 , and 4.0 out of 5 in 2016, 2017, and 2018, respectively) across all 3 years.

\section{Adoption}

Approximately $12 \%(n=28)$ of the program participants responded to the online follow-up survey. Approximately $50 \%(n=14)$ of the online follow-up survey respondents participated for the first time in 2016, 36\% $(n=10)$ in 2017, and the remaining 14\% $(n=4)$ in 2018. Among the online follow-up survey respondents, $60.7 \%$ 
Table 4 Effects of ultrasound education program for obstetric, fetal, neonatal and congenital anomaly in Nepal, 2016, 2017, and 2018 on self-confidence, knowledge, and satisfaction

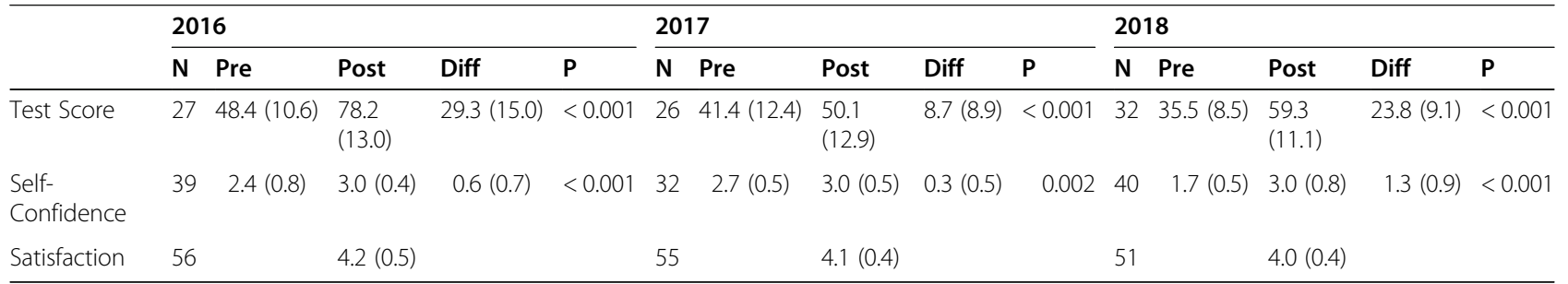

( $n=17)$ indicated that they were currently using ultrasound in their daily practice (Table 5). When we divided the respondents by specialty, we found that most of the radiologists $(75 \%, n=3)$ and other specialties $(71.4 \%, \mathrm{n}=$ $10)$ and half of OB-GYNs $(50 \%, n=4)$ began to use ultrasound in their daily practice after participating in the education program. None of the pediatricians who participated in the survey have reported the use of ultrasound.

Table 6 illustrates the in-depth interview results including major limitations of ultrasound utilization. The lack of availability of ultrasound machines and lower competency levels due to lack of regular training and practice were identified as the major challenges for the adoption of ultrasound. Moreover, restrictive institutional and national policies were also identified as

Table 5 Online follow-up survey results regarding ultrasound usage

\begin{tabular}{ll}
\hline Using Ultrasound in Current Practice & $\mathbf{N = 2 8}(\mathbf{\%})$ \\
\hline Overall (all subgroups) & $17(60.71)$ \\
Radiologist & $3(75.00)$ \\
OB-GYN & $4(50.00)$ \\
Pediatrician & $0(0.00)$ \\
Others & $10(71.42)$ \\
Changed Practice after Training Program & \\
Yes & $14(82.35)$ \\
No & $3(17.65)$ \\
Techniques Currently Used & \\
Fetal CNS & $10(58.82)$ \\
Fetal Doppler & $8(47.05)$ \\
Fetal echo & $3(17.65)$ \\
Fetal thorax & $1(5.89)$ \\
Biometry & $2(11.76)$ \\
Cervical length & $2(11.76)$ \\
Fetal abdomen & $2(11.76)$ \\
Genetic sonography & $0(0)$ \\
Pediatric echo & $0(0)$ \\
Fetal genitourinary & $0(0)$ \\
\hline OB-GYN: obstetrician-gynecologist & \\
& \\
\hline
\end{tabular}

"OB-GYN: obstetrician-gynecologist limitations. Radiologists performed ultrasound in their daily practice, whereas non-radiologists used ultrasound during emergencies and for confirmation of their own diagnosis. Some non-radiologists from institutions that promote the use of ultrasound reported performing a high volume of ultrasound in their practice after participating in the education program. Fetal neurosonogram and fetal Doppler techniques were widely used (58.8 and $47.1 \%$ of ultrasound users, respectively), whereas genetic sonography, pediatric echo, and fetal genitourinary techniques have not been used. A non-radiologist expressed concerns on overuse of ultrasound, "There is a high dependency on ultrasound, leading to ultrasound overuse, in conditions where other diagnostics methods were sufficient." A radiologist also had a concern regarding ultrasound misuse as "ultrasound can be used for commercial purposes."

All the interviewees expressed positive attitudes toward the education program. They attributed the program participation to the use of ultrasound in their workplaces. They also expressed that the program helped them improve their use of ultrasound in their practice. For future direction of the program, the interviewees suggested that certification and accreditation policy, educational session on the misuse of ultrasound, and online follow-up after the program should be implemented. A radiologist expressed, "...there is no system of accreditation. It encourages using ultrasound without any proper certification. There is potential misuse of ultrasound. There is no proper follow up."

\section{Implementation}

The program was developed and implemented in collaboration with the DHKUH and Nepal Society of Obstetricians and Gynaecologists. The Ultrasound Society of Nepal joined as a co-organizer, whereas the Nepal Radiologists' Association and Nepal Paediatric Society endorsed and promoted the education program. The Nepal Medical Association accredited the continuing medical education credits based on curriculum and content. The involvement of Nepal medical societies and associations ensured a wide acceptance of the program. 
Table 6 Themes, subthemes, and quotations for adoption and maintenance dimensions

\begin{tabular}{lcc}
\hline Themes and Definitions & Subthemes and Definitions & Quotes
\end{tabular}

\section{Ultrasound use:}

Participants expressed they were using ultrasound at least at some level in all targeted specialties (OB-GYN, pediatrician, and radiologist), independently or with support

from the radiology department

\section{Process/events:}

General practice of ultrasound and occasions of

use in the participant's institution
Perspective toward educational program: Respondents expressed their views and observations regarding the educational program and changes attributed to the program

\section{Frequency:}

Number of cases participants directly perform ultrasound

Use of ultrasound in regular practice: Use of ultrasound in regular practice attributed to the program

\section{Correction in practice:}

Previously used techniques were corrected or improved based on the program

\section{Negative perspectives:}

The negative views toward the program and its potential negative outcomes

Lack of ultrasound machine: Participants mentioned that there are no ultrasound machines or fewer ultrasound machines
"... In our institution, routine ultrasound is performed by the radiologist. However, for screening purposes such as emergency ultrasound, it has been performed by other healthcare workers in other departments including emergency department and obstetrics and gynecology department" - Pediatrician

"To determine early pregnancy, the general condition of the fetus during late pregnancy, the appropriate location of the placenta, the alignment of the uterus, ultrasound in gynecology is required. Moreover, to confirm the presence of tumors and fibrocystic lesions or latencies, performing ultrasound is an essential part of our practice." - OB-GYN

"Cranial ultrasound, regular echocardiography, and abdominal ultrasound including renal scan are performed regularly to screen and to diagnose more complicated cases and to immediately respond to emergency department and intensive care unit care" - Pediatrician

“....Hence possibly in a day we examine approximately 55 patients; among them, I examine possibly 10 patients daily..." - OB-GYN "I perform ultrasound in nearly $50 \%$ of admitted cases and 10 to $20 \%$ of outpatient department cases" $^{\prime \prime}$-Pediatrician

"In the past, ultrasonography and sonography were considered as the standard modalities, but they were mostly unavailable. However, today, these modalities are regularly performed in clinical practice in wards, outpatient departments, and even in the emergency department. It has been well accepted by the patients as well." - OB-GYN

"...nowadays, everyone is competent to perform ultrasound regularly. Moreover, healthcare workers already decide on the dose of inotropes based on echo results." - Pediatrician

"...the training has addressed the topics we dealt in daily practice, improving confidence in establishing clinical decisions..." - OB-GYN

"The ultrasound was already used during the past years, but specific guidelines in performing ultrasound to assess congenital anomalies were not yet established... after attending the course, we already properly performed ultrasonography. The knowledge obtained was shared with the resident students as well, and they significantly improved." - OB-GYN

"...This type of program has several controversies, specifically in Nepal...there is no system of accreditation. It encourages the use of ultrasound without any proper certification. There is potential misuse of ultrasound. There is no proper follow-up." - Radiologist

"The gynecologists are not performing ultrasonography in their clinics because of the unavailability of any ultrasound machine, including my clinic. In private hospital or clinic setup, it is expensive to keep an ultrasound machine." - OB-GYN

"Initially, lack of ultrasound equipment was one 
Table 6 Themes, subthemes, and quotations for adoption and maintenance dimensions (Continued)

Subthemes and Definitions

\section{Lower competency:}

Participants expressed low competency and nonconfidence in performing ultrasound on their own

Perspective on ultrasound use: How individual observed the use of ultrasound in the current context

\section{Overuse of ultrasound:}

Use of ultrasound where it was not absolutely required. High dependency on the ultrasound giving lower importance to other aspects

Misuse of ultrasound:

Use of ultrasound guided other intentions

Important diagnostic tools:

Participants express ultrasound as the important tool for diagnosis and investigation

Improved quality of patient management: Participants associate ultrasound use with improved patient management

\section{Quotes}

of the challenges; however, currently, it is now gradually addressed; for instance, the Seoul National University donated a decent echo machine, and we are significantly grateful for that. We are using this machine for pediatric echocardiography and transcranial scans. Gradually, our department is also equipped with few other machines." -Pediatrician

"Every single day, one doctor has to perform 10 to 15 congenital anomaly scans; hence, it is a difficult job. If the cases were significantly few, that is, 4 to 5 per day, it would have been covered by the current infrastructure." Radiologist

"During emergency conditions in high-risk pregnancy, OB-GYNshould be able to perform ultrasonography, but considering the insufficient training programs for OB-GYN, performing ultrasonography is the major cause of OB-GYN's lower competencies." - OB-GYN

"We over diagnosed several diseases because of ultrasound. However, sometimes, we try to overlook our clinical practice and our clinical skills similar to what we do when using the stethoscope and when performing palpitations, and our clinical practice is sometimes insufficient, and we depend on the ultrasound machines. Hence, I think that sometimes we have to assess the risks and benefits. It has changed our decision-making skills." - Pediatrician

"The use of ultrasound for inappropriate cases and for commercial purposes. It is easy to obtain 1000 rupee if you use ultrasound. People undergo 15 days of training and can subsequently open their ultrasound service center. This is considered a significant unethical issue." - Radiologist

"Ultrasound is one of the most important diagnostic or investigative tools."

"When performing clinical practice and when using the ultrasound machines to confirm our clinical diagnosis, disease diagnosis and management is significantly easier and accurate. Hence, I think ultrasound machine has changed our decision-making skills and improve our patient care." - Pediatrician

Maintenance: Institution have policies (also includes national), infrastructures, and initiatives to sustain the use of ultrasound

Infrastructure maintenance:

The participants expressed recent developments or improvements in instruments, spaces, and human resource empowerment
Availability of ultrasound machines: Participants mentioned about the upgrade or addition of ultrasound machines

Competency of human resources: Participants mentioned the availability of trained human resources or doctors who can perform ultrasound and opportunity for getting human resources trained
"In Nepal, we have a relatively good number of ultrasound machines that are available not only in hospital but also in outreach centers such as in remote areas. For the past 2 years, I think we added 20 ultrasound machines." -Radiologist "Our hospital has provided ultrasound machines in our wards, labor rooms, emergency rooms, and in all areas where we might need an ultrasound machine." - OB-GYN

"....the consultants and junior doctors in my department are competent in performing basic ultrasonography by themselves." - OB-GYN "Sometimes, when we encountered difficult cases, we used to discuss with healthcare professors who were able to undergo trainings from Korea via emails and discuss the ultrasound 
Table 6 Themes, subthemes, and quotations for adoption and maintenance dimensions (Continued)

Themes and Definitions
Policy:
The participants expressed national or
institutional policy including formal written
policy or informal practice-related policy

policy or informal practice-related policy

\section{Subthemes and Definitions}

Institutional policy to encourage practice:

Participants mentioned about written policy or unwritten but understandable policy that encourages the use of ultrasound

\section{Quotes}

images." - Pediatrician

"There is no written protocol regarding the use of ultrasound, but limitations regarding its use are not yet established. When we train the resident doctors, they perform the ultrasound scan for inpatient children during the evening whenever they are free." - Pediatrician "There is no written guideline stating that ultrasonography should be performed by a gynecologist, but in the department, the use of ultrasound is encouraged. During the department meeting, we encourage colleagues to perform ultrasonography to establish the diagnosis of a disease." - OB-GYN

"When I joined the residence here, a consultant doctor (teacher) said that I will be doing transvaginal sonography gradually and will be diagnosing the intrauterine pregnancy." - OBGYN

Restriction in practice by policy: Participants mentioned about restriction in use of ultrasound in practice or incorporation in formal guidelines by policy
"We do not have any written (formal) document indicating ultrasound scans need to be performed by obstetricians, but as part of our examinations, we perform it." - OB-GYN "The eligibility of a healthcare worker to perform ultrasound and the guidelines used to certify a healthcare worker regarding the use of ultrasound are not yet established in Nepal. Currently, any healthcare worker (including staff nurse, health assistant, and even Ayurvedic doctor) with an ultrasound machine can perform ultrasonography. There is no proper system of accreditation." - Radiologist

"My findings will not be as strong as it is when evaluated by a radiologist for documentation, even if we can identify the problem, we need to refer our findings to a radiologist." - OB-GYN

"An ultrasound training center had been established in Dhulikhel Hospital with good infrastructures. Separate department had been established to handle this. The institution has started the ultrasound training on their own and also has expanded their collaborations. The curriculums and modules for various levels of ultrasound education had been developed, and education is being provided continuously." Radiologist
Figure 1 shows the process of implementation. The program had four major components: lectures, case discussion and experience sharing, live demonstration, and program evaluation (Fig. 1). The major difference in the program implementation across the years was the exclusion of live demonstration sessions in 2018 based on the feedback and logistic difficulties. Furthermore, major differences in the program implementation modality were not observed.

\section{Maintenance}

The Interviewees mentioned that ultrasound use is still lagging due to unclear policy and high cost for the ultrasound machines for private hospitals (Table 6). Few of the institutions have developed infrastructure such as purchasing ultrasound machines and trained human resources and fostered networks for the discussion of cases via tele chats. Both radiologists and non-radiologists expressed dissatisfaction with the current policy in terms of ambiguity in accreditation and the absences of practical guidelines for ultrasound use by non-radiologist. A radiologist expressed, "The eligibility of a healthcare worker to perform ultrasound and the guidelines used to certify a healthcare worker regarding the use of ultrasound are not yet established in Nepal. Currently, any healthcare worker (including staff nurse, health assistant, and even Ayurvedic doctor) with an ultrasound machine can perform ultrasonography. There is no proper system 


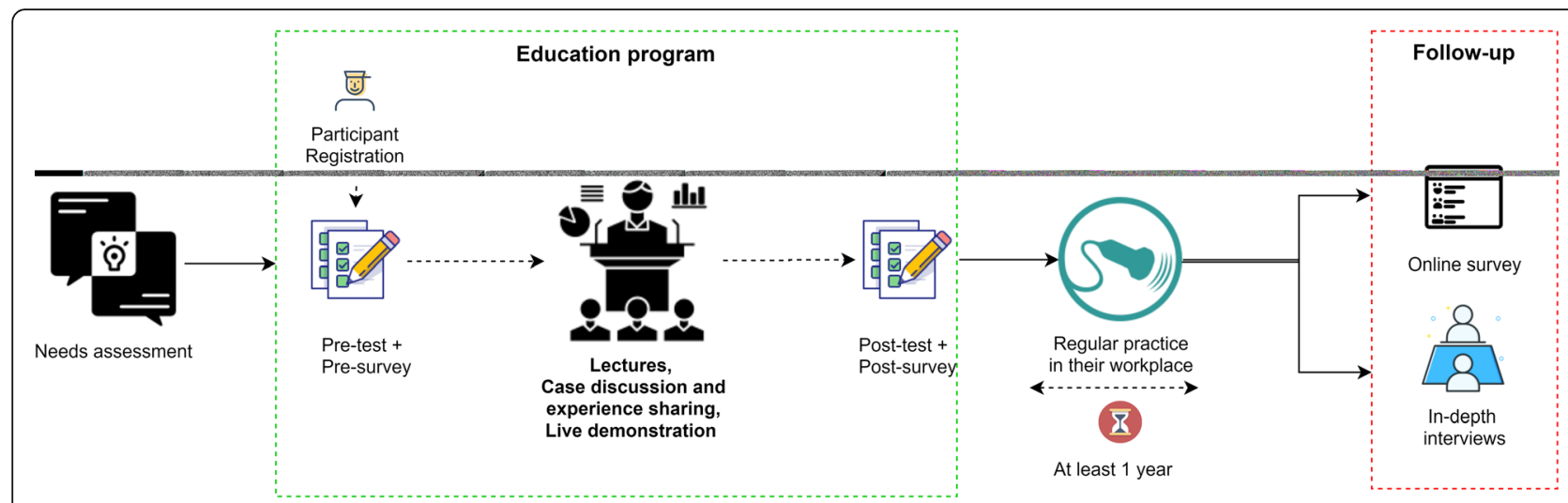

Fig. 1 Process of the implementation and evaluation of the education program

of accreditation." Similarly, a non-radiologist practicing ultrasound expressed, "My findings will not be as strong as it is when evaluated by a radiologist for documentation. Even if we can identify the problem, we need to refer our findings to a radiologist." These types of ambiguity and inefficiency in national policy on accreditation was expressed as the major limitation of ultrasound utilization by non-radiologists. The high cost of an ultrasound device makes it difficult for private hospitals to use ultrasound prohibiting them to practice and maintain the skills that they have learned. Only one institution established an ultrasound-training center and initiated regular training for doctors and healthcare workers, partly stimulated by the education program.

\section{Discussion}

To improve the ultrasound utilization and the service quality of maternal and child health in Nepal, the JW LEE CGM and the DHKUH have conducted "The Ultrasound, CT and MRI Education Program for Obstetric, Fetal, Neonatal, and Congenital Anomaly" in collaboration with the Nepali Professional Societies. This study

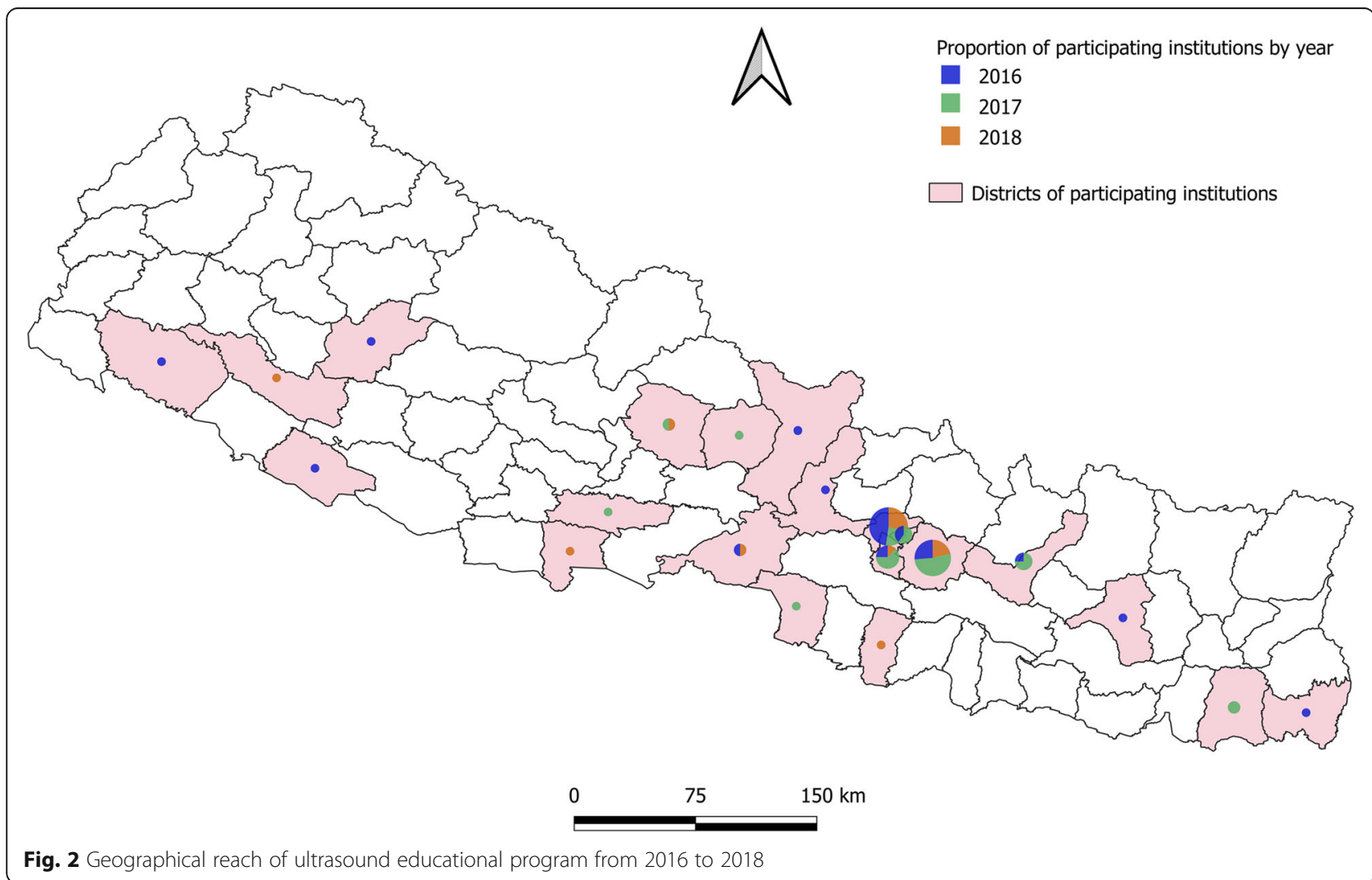


evaluated the 2-day intensive ultrasound education program conducted in Nepal from 2016 to 2018 in terms of its Reach, Effectiveness, Adoption, Implementation, and Maintenance using the RE-AIM framework. This study showed that the ultrasound education program was effective in improving the knowledge and self-confidence of physician and non-physician participants. However, only half of the online survey respondents indicated that they used learned ultrasound techniques in their daily practices after participating in the education program. This study also observed significant heterogeneity in ultrasound utilization by site and across all healthcare professions in Nepal. Changing regulations at institutional and national levels to facilitate use of ultrasound, and improve availability of ultrasound devices may increase the adoption and maintenance of the ultrasound in practice.

The 'Reach' dimensions of the program were satisfactory in terms of participants' diversity in specialty and affiliation. Geographically, however, the program reached approximately $27 \%$ of the districts in Nepal. This may be due to the uneven distribution of ultrasound services across healthcare facilities and providers in Nepal. Unavailability of machines and human resources might have resulted in lower geographical coverage. A previous study on the availability of technology for trauma care in Nepal, which accessed 56 small hospitals (with a bed capacity of 30 to 100) and 29 large hospitals, reported that ultrasound was not available in any of accessed small hospitals [19]. The health facility survey in 2015 showed that only $21.1 \%$ of the district-level hospitals had basic equipment and approximately half of the sanctioned position of medical officers and Doctor of Medicine in General Practice (MD-GP) were completed [12].

This study shows that the program was effective in increasing the healthcare workers' knowledge and selfconfidence in using ultrasound. The participants were also satisfied with this program. We observed a slight decrease in the satisfaction score from 2016 to 2018, but the mean scores were above 4 out of 5 . The education program on ultrasound with several implementation modalities and durations with the use of pre- and post-test to assess knowledge also resulted in significant improvement in knowledge immediately after the programs [9, $13,22,23]$. A previous study from Nepal on point-ofcare ultrasound training also reported improvement in post-test scores in the 1-day training, which was mostly participated by doctors without prior experience [13]. Further, Shaffer et al. (2017) reported that the selfreported confidence for four specific skills increased among the participants at the end of the short-term (5day) training program on bedside emergency ultrasound [24]. We removed the live demonstration component in
2018 due to technical and logistical difficulties, but it did not lead to a significant decrease in the three components of the 'Effectiveness' dimension.

This study found a mid-level (60\%) of adoption at the individual level via online follow-up survey. The nonadoptees emphasized that further policy changes to encourage the use of ultrasound by non-radiologists along with infrastructure development are considered the major limitations in the use of ultrasound. Setting up and maintenance of the machines can also be significantly challenging in rural areas of Nepal [11]. Another interviewee emphasized the uncertainty in the current policies, implying the prohibitive use of ultrasound by the non-radiologists. The clinical guidelines do not also explicitly mention the protocols regarding the use of ultrasound by doctors in the non-radiology departments. A clear scope on the use of ultrasound and eligibility of performance may facilitate ultrasound utilization and service quality.

The institutional-level 'Maintenance' dimension of the RE-AIM framework had mixed results. Despite the challenges and limitations regarding the changes in policy and infrastructure development, one of the participating institutions in Nepal had established an ultrasound training school, and they collaborated with different specialties to develop courses for training physician and non-physician healthcare providers. However, some organizations are still having trouble in formulating clear institutional policies on ultrasound use by non-radiology doctors. The establishment of the ultrasound training center can be considered a crucial step in the sustainability of interdisciplinary educational programs.

This study has several limitations. First, we used convenience sampling methods for in-depth interviews because of time constraints and insufficient resources to travel beyond Kathmandu and its vicinity. The second limitation was low participation rates in evaluations. Participation in the evaluation was voluntary, and many of the registered participants were allowed to select sessions and skip tests and surveys. We offered flexibility since the second day of the training took place during the workday. Many participants were not able to arrange a leave for the day. In the case of the online survey, a low response rate was expected, thus we tried to mitigate this by sending three reminder emails. Due to low participation rates, the findings may have induced selection bias. Finally, the evaluation of self-confidence and use of ultrasound was based on self-reporting, which may cause social desirability bias. Despite these limitations, this study provides evidence that the education program can be used as a platform for sharing knowledge and experiences across healthcare providers with diverse backgrounds. 


\section{Conclusion}

Our study found that the ultrasound education program effectively improved the knowledge and self-confidence of participants in practicing ultrasound. The program also had a positive impact on ultrasound utilization by encouraging participants to use ultrasound; however, determining the influencing institutional environment that facilitates ultrasound utilization and improves service quality was still considered challenging due to the current regulations. Future studies are required to comprehensively understand the various elements influencing the use of learned knowledge and acquired skills that translates into actual practice that significantly affects the health at the population level.

\section{Abbreviations \\ LMICs: Low- and Middle-income Countries; WHO: World Health Organization; JW LEE CGM: JW LEE Center for Global Medicine at Seoul National University College of Medicine; RE-AIM: Reach, Effectiveness, Adoption, Implementation, and Maintenance; DHKUH: Dhulikhel Hospital Kathmandu University Hospital; OB-GYN: Obstetrician-gynecologist; CT: Computed Tomography; \\ MRI: Magnetic Resonance Imaging; RQDA: R Qualitative Data Analysis; NGO: Non-Governmental Organization; SNUH: Seoul National University Hospital in the Republic of Korea; MD-GP: Doctor of Medicine in General Practice}

\section{Acknowledgements}

We would like to thank Raphael International, an NGO of the Republic of Korea, for providing administrative support in organizing the program from 2016 to 2017. We would also like to thank the Nepali Professional Societies for supporting and promoting the educational program.

\section{Authors' contributions}

WK and JK conceptualized the study. JK and PRS collected, cleaned and analyzed the data. WK, JK, PRS, SC, and JH were major contributors in writing the manuscripts. JSP and SRT analyzed the curriculum and contributed to the writing technicalities of ultrasound parts. All authors reviewed and provided critical comments. All authors have access to the data. The author(s) read and approved the final manuscript.

\section{Funding}

This research was financially supported by the JW LEE Center for Global Medicine of Seoul National University College of Medicine, Seoul, Republic of Korea.

\section{Availability of data and materials}

The datasets generated and/or analyzed during this study are available from the corresponding author upon reasonable request.

\section{Ethics approval and consent to participate}

All methods were carried out in accordance with relevant guidelines and regulations. Participants provided verbal and/or written consent. The study protocol was reviewed and obtained ethical approval from Institutional Review Boards at the Seoul National University Hospital in the Republic of Korea (SNUH IRB No. 1906-036-1038).

\section{Consent for publication}

Not applicable.

\section{Competing interests}

The authors declare that they have no conflict of interest to disclose.

\section{Author details}

${ }^{1}$ JW LEE Center for Global Medicine, Seoul National University College of Medicine, Seoul, Republic of Korea. ${ }^{2}$ Department of Community Program, Dhulikhel Hospital-Kathmandu University Hospital, Kavre, Nepal. ${ }^{3}$ Department of Health Law, Policy \& Management, Boston University School of Public
Health, Boston, MA, USA. ${ }^{4}$ Department of Obstetrics and Gynecology, Seoul National University College of Medicine, Seoul, Republic of Korea. ${ }^{5}$ Department of Obstetrics and Gynecology, Dhulikhel Hospital-Kathmandu University Hospital, Kavre, Nepal. ${ }^{6}$ National Assembly Futures Institute, Seoul, Republic of Korea. 'Department of Thoracic and Cardiovascular Surgery, Seoul National University College of Medicine, Seoul, Republic of Korea.

Received: 16 September 2020 Accepted: 17 December 2020

Published online: 15 January 2021

\section{References}

1. WHO. Birth Defects In South-east Asia A Public Health Challenge. 2013. http://apps.searo.who.int/PDS_DOCS/B4962.pdf.

2. Bhandari S, Sayami JT, Raj R, Banjara MR. Prevalence of congenital defects including selected neural tube defects in Nepal: results from a health survey. BMC Pediatr. 2015. https://doi.org/10.1186/s12887-015-0453-1.

3. Ansari I, Rajbhandari R, Chilise S, Shalh G, Maskey P, Maharjan R, et al. Congenital malformations at birth in 7,922 consecutive deliveries at Patan Hospital, Nepal. J Patan Acad Heal Sci. 2015;1:4-7. https://doi.org/10.3126/ jpahs.v1i2.16636

4. Baral G. Birth Defects: Emerging Public Health Issue. J Nepal Health Res Counc. 2015;:13.

5. Todros T, Capuzzo E, Gaglioti P. Prenatal diagnosis of congenital anomalies. Images Paediatr Cardiol. 2001;3:3-18. http://www.ncbi.nlm.nih.gov/pubmed/ 22368596.

6. Sippel S, Muruganandan K, Levine A, Shah S. Review article: Use of ultrasound in the developing world. Int J Emerg Med. 2011;4.

7. WHO. WHO Recommendations on Antenatal Care for a Positive Pregnancy Experience: Ultrasound Examination. 2016. https://apps.who.int/iris/ bitstream/handle/10665/259946/WHO-RHR-18.01-eng.pdf? sequence=1. Accessed 31 Jan 2020.

8. Nathan R, Swanson JO, Marks W, Goldsmith N, Vance C, Sserwanga NB, et al Screening obstetric ultrasound training for a 5-country cluster randomized controlled trial. Ultrasound Q. 2014;30:262-6. https://doi.org/10.1097/RUQ. 0000000000000096

9. Lagrone LN, Sadasivam V, Kushner AL, Groen RS. A review of training opportunities for ultrasonography in low and middle income countries. Trop Med Int Heal. 2012;17:808-19.

10. Subedi KS, Sharma P. Development of Radiology in Nepal: Gearing Up for Mountainous Challenges. J Am Coll Radiol. 2013;10:291-5. https://doi.org/10. 1016/j.jacr.2012.12.020.

11. Filkins M, Halliday S, Daniels B, Bista R, Thapa S, Schwarz R, et al. Implementing Diagnostic Imaging Services in a Rural Setting of Extreme Poverty: Five Years of X-ray and Ultrasound Service Delivery in Achham, Nepal. J Glob Radiol. 2015;1. https://doi.org/10.7191/jgr.2015.1001.

12. Ministry of Health-Nepal. Nepal Health Facility Survey 2015. Kathmandu; 2016. https://dhsprogram.com/pubs/pdf/SPA24/SPA24.pdf.

13. Thapa A (Kathmandu MCT hospital), KC B, Shakya B. Effect of short courses on perception to use of ultrasound in trauma and ICU settings. 2017;6:102-109.

14. Bentley S, Hexom B, Nelson BP. Evaluation of an Obstetric Ultrasound Curriculum for Midwives in Liberia. J Ultrasound Med. 2015;34:1563-8. https://doi.org/10.7863/ultra.15.14.08017.

15. Glasgow RE, Vogt TM, Boles SM. Evaluating the public health impact of health promotion interventions: the RE-AIM framework. Am J Public Health. 1999;89:1322-7. https://doi.org/10.2105/ajph.89.9.1322.

16. Glasgow RE, Harden SM, Gaglio B, Rabin B, Smith ML, Porter GC, et al. REAIM Planning and Evaluation Framework: Adapting to New Science and Practice With a 20-Year Review. Front public Heal. 2019;7:64. https://doi.org/ 10.3389/fpubh.2019.00064.

17. Holtrop JS, Rabin BA, Glasgow RE. Qualitative approaches to use of the REAIM framework: rationale and methods. BMC Health Serv Res. 2018;18:177. https://doi.org/10.1186/s12913-018-2938-8.

18. Sweet SN, Michalovic E, Baxter K, Baron J, Gainforth HL. RE-AIMing conferences: a reach, effectiveness, and maintenance evaluation of the Rick Hansen Institute's Praxis 2016. Transl Behav Med. 2019. https://doi.org/10. 1093/tbm/ibz002.

19. Shah MT, Bhattarai S, Lamichhane $N$, Joshi $A$, LaBarre $P$, Joshipura M, et al. Assessment of the availability of technology for trauma care in Nepal. Injury. 2015:46:1712-9. https://doi.org/10.1016/J.INJURY.2015.06.012.

20. Ronggui H. RQDA: R-based Qualitative Data Analysis. 2016. 
21. Corbin J, Strauss A. Grounded Theory Research: Procedures, Canons, and Evaluative Criteria. Qual Sociol. 1990;13 https://link.springer.com/content/ pdf/10.1007\%2FBF00988593.pdf.

22. Sweet SN, Ginis KAM, Estabrooks PA, Latimer-Cheung AE. Operationalizing the RE-AIM framework to evaluate the impact of multi-sector partnerships. Implement Sci. 2014;9:74. https://doi.org/10.1186/1748-5908-9-74.

23. Pietersen PI, Madsen KR, Graumann O, Konge L, Nielsen BU, Laursen CB. Lung ultrasound training: a systematic review of published literature in clinical lung ultrasound training. Crit Ultrasound J. 2018;10:23. https://doi. org/10.1186/s13089-018-0103-6.

24. Shaffer M, Brown HA, McCoy C, Bashaka P. Evaluation of a Short-term Training Program in Bedside Emergency Ultrasound in Southwestern Tanzania. J Ultrasound Med. 2017;36:515-21. https://doi.org/10.7863/ultra.16. 04006.

\section{Publisher's Note}

Springer Nature remains neutral with regard to jurisdictional claims in published maps and institutional affiliations.

Ready to submit your research? Choose BMC and benefit from:

- fast, convenient online submission

- thorough peer review by experienced researchers in your field

- rapid publication on acceptance

- support for research data, including large and complex data types

- gold Open Access which fosters wider collaboration and increased citations

- maximum visibility for your research: over $100 \mathrm{M}$ website views per year

At $\mathrm{BMC}$, research is always in progress.

Learn more biomedcentral.com/submissions 